\title{
Free Electron Relativistic Correction Factors to Collisional Excitation and Ionisation Rates in a Plasma
}

\author{
J.J. Beesley ${ }^{\mathrm{a}, *}$, S.J. Rose ${ }^{\mathrm{a}}$ \\ ${ }^{a}$ Plasma Physics Group, Imperial College, Blackett Laboratory, Prince Consort Road, \\ London SQ7 2AZ, UK
}

\begin{abstract}
This paper presents approximate correction factors to collisional excitation and ionisation rates which account for special relativity in the free electron motion as a function of free electron temperature and the threshold energy of the reaction. These are calculated by taking the ratio of collisional rates derived from simple empirical cross sections using relativistic and non-relativistic mechanics. These results are extended to de-excitation and three body recombination rates using detailed balance. It is found that the relativistic correction is significant in regimes potentially important to galactic intracluster media and diagnostic dopants in burning ICF plasmas.
\end{abstract}

Keywords: ionisation rates, excitation rates, relativity, correction factors, collisional rates

\section{Introduction}

When calculating rates of collisional excitation and ionisation the free electrons in a plasma are usually taken to follow the Maxwell-Boltzmann distribution [1]. This means that they are treated classically, and hence is valid as an approximation when both quantum mechanical and relativistic effects are insignificant. The quantum mechanical degenerate limit has been well studied

\footnotetext{
* Corresponding author

Email addresses: jonathan.beesley17@imperial.ac.uk (J.J. Beesley), s.rose@imperial.ac.uk (S.J. Rose)
}

Preprint submitted to Elsevier

September 20, 2019 
and correction factors to excitation and ionisation rates accounting for quantum effects have been calculated in some detail [2] [3]. The relativistic limit has been comparatively neglected. There are, though, good reasons to pay attention to it: in the context of laboratory plasmas Tabak [4] predicts that burning D plasmas in ICF could reach bulk reduced temperatures of up to $\theta=0.31$, and Xenon and Krypton dopants (which should remain only partially ionised up to such temperatures) are regularly included in ICF as diagnostics [5] [6] . In the astrophysical context, the intracluster medium can reach temperatures above $\theta=0.04$ [7]. Depending, of course, on the accuracy needed, relativistic corrections could be significant in both cases. Note we are here defining the "reduced temperature" by

$$
\theta=k_{B} T / m c^{2}
$$

with $\mathrm{T}$ temperature, $\mathrm{m}$ the electron mass.

The main correction to be made when accounting for relativistic effects is to replace the Maxwell-Boltzmann distribution,

$$
f_{M B}(\eta, \theta)=2 \sqrt{\frac{\eta}{\pi}} \theta^{-\frac{3}{2}} e^{-\frac{\eta}{\theta}},
$$

(written here in terms of $\eta=E_{K} /\left(m c^{2}\right)$, the reduced electron kinetic energy) with the Maxwell-Jüttner [8] distribution,

$$
f_{M J}(\gamma, \theta) \equiv \frac{\gamma^{2} \beta}{\theta K_{2}(1 / \theta)} e^{-\frac{\gamma}{\theta}}
$$

where $\beta=\frac{v}{c}, \gamma=1 / \sqrt{1-\beta^{2}}=\eta+1$ and $K_{2}$ is the modified Bessel function of the second kind. The two are plotted in figure 1 for several temperatures. The Maxwell-Boltzmann distribution is recovered from the Maxwell-Jüttner in the expected classical limits $\theta \rightarrow 0$ and $\gamma \rightarrow 1$. Previous authors [9] [10] [11] have used these distributions to find a simple generic "relativistic correction factor" to excitation and ionisation rates based on the ratio of free electron partition functions (see Sect. 2, equation 13). In this paper we develop more 

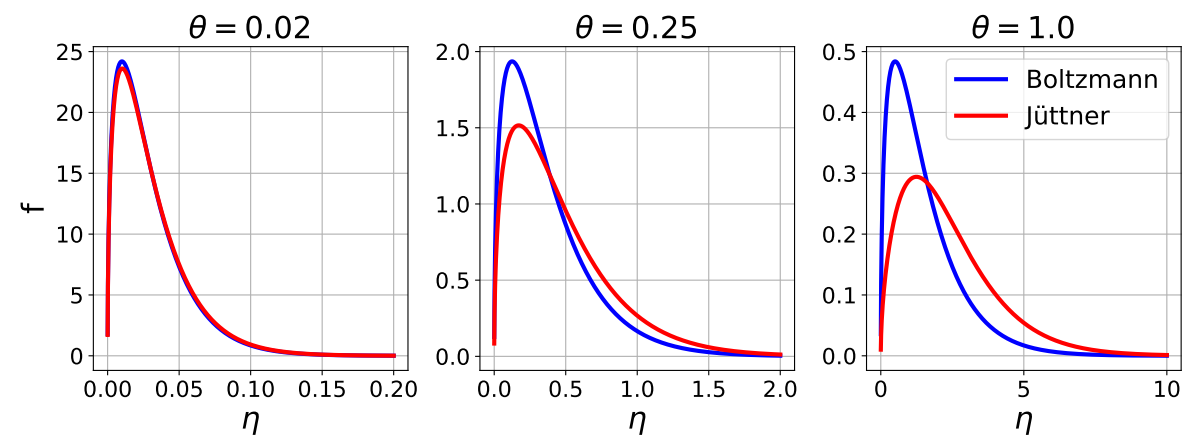

Figure 1: Maxwell-Boltzmann and Maxwell-Jüttner distributions plotted against reduced kinetic energy for a range of temperatures. Classically, energy is everywhere quadratic in momentum, while relativistically it becomes linear at high momentum. Therefore the density of energy states in phase space is classically suppressed at high momentum while relativistically it approaches a constant, so the normalised classical distribution is, compared to the relativistic, skewed towards low energies.

sophisticated correction factors by considering simple cross-sections for excitation and ionisation due, respectively, to Van Regemorter [12] and Lotz [13]. We also use detailed balance to extend these results to de-excitation and three-body recombination rates.

\section{Calculation of Rates}

\subsection{Method}

A collisional rate per electron per ion can be written in the form, keeping the cross section and energy distribution general,

$$
r\left(\eta_{T}, \theta\right)=\int_{\eta_{T}}^{\infty} \sigma\left(\eta / \eta_{T}\right) f(\eta, \theta) v(\eta) d \eta .
$$

where $v(\eta)$ is the velocity, $f(\eta, \theta)$ is the probability of finding a given electron at energy $\eta, \eta_{T}$ is defined by

$$
\eta_{T}=E_{T} / m c^{2}
$$


where $E_{T}$ is the threshold transition energy, and $\sigma\left(\eta / \eta_{T}\right)$ is the cross section of the ions for the relevant collision reaction with the free electrons, which we are treating as a function of $\eta / \eta_{T}$ (as is the case for most simple empirical or semi-empirical formulas for the ionisation or excitation cross-sections). It has the property $\sigma(x<1)=0$.

The rate of collision per free electron for the two cases is then

$$
\begin{aligned}
& r_{M B}\left(\eta_{T}, \theta\right)=\int_{\eta_{T}}^{\infty} \sigma\left(\eta / \eta_{T}\right) v_{c}(\eta) f_{M B}(\eta, \theta) d \eta, \\
& r_{M J}\left(\eta_{T}, \theta\right)=\int_{\eta_{T}}^{\infty} \sigma\left(\eta / \eta_{T}\right) v_{r}(\eta) f_{M J}(\eta, \theta) d \eta,
\end{aligned}
$$

where we are using the $\mathrm{r}$ and c subscript on the velocity to denote, respectively, the relativistic and classical expressions for velocity in terms of kinetic energy. The quantity we want to calculate is the correction factor due to relativity,

$$
R\left(\eta_{T}, \theta\right) \equiv \frac{r_{M J}\left(\eta_{T}, \theta\right)}{r_{M B}\left(\eta_{T}, \theta\right)} .
$$

For the relativistic case

$$
f_{M J}(\eta, \theta) v_{r}(\eta)=\frac{2 c e^{-\frac{1}{\theta}}}{\theta K_{2}\left(\frac{1}{\theta}\right)}\left(\eta+\frac{1}{2} \eta^{2}\right) e^{-\frac{\eta}{\theta}} .
$$

where $\beta=\frac{v}{c}, \gamma=1 / \sqrt{1-\beta^{2}}=\eta+1$. For the classical case

$$
f_{M B}(\eta, \theta) v_{c}(\eta)=2 c \sqrt{\frac{2}{\pi}} \theta^{-\frac{3}{2}} \eta e^{-\frac{\eta}{\theta}} .
$$

Therefore the correction factor (8) can be written

$$
R\left(\eta_{T}, \theta\right)=\Theta(\theta) \cdot\left[1+\frac{\theta}{2} P\left[\sigma ; \frac{\eta_{T}}{\theta}\right]\right]
$$

where we have defined the functional, using the substitution $z=\eta / \theta$,

$$
P\left[\sigma ; \frac{\eta_{T}}{\theta}\right] \equiv \frac{\int_{\eta_{T} / \theta}^{\infty} z^{2} \sigma\left(z \frac{\theta}{\eta_{T}}\right) e^{-z} d z}{\int_{\eta_{T} / \theta}^{\infty} z \sigma\left(z \frac{\theta}{\eta_{T}}\right) e^{-z} d z}
$$




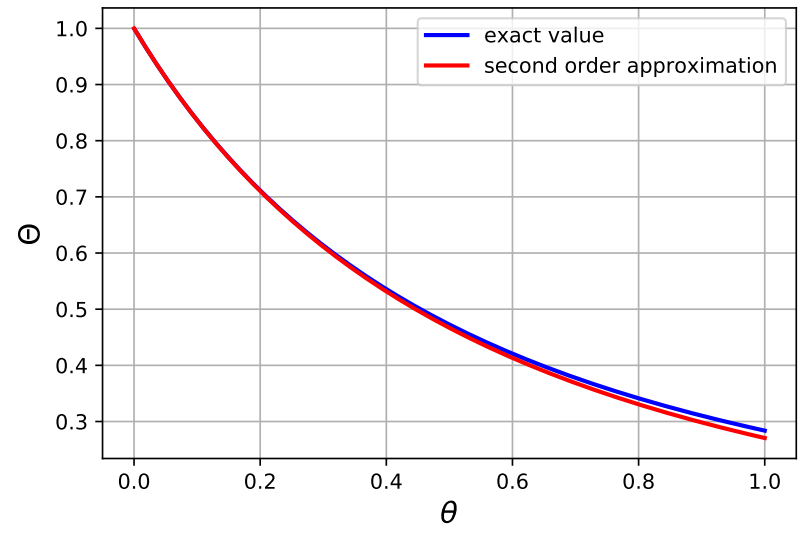

Figure 2: General temperature-dependent prefactor to relativistic correction factor of collisional rates. Both the exact value and second-order approximate value are plotted, showing very little difference between the two.

and the temperature-dependent prefactor is given by

$$
\Theta(\theta) \equiv \sqrt{\frac{\pi}{2}}\left[\frac{\sqrt{\theta} e^{-\frac{1}{\theta}}}{K_{2}\left(\frac{1}{\theta}\right)}\right]=\frac{Z_{M B}(\theta)}{Z_{M J}(\theta)}
$$

where $Z_{M B / M J}$ is the partition function for a single free particle in the classical/relativistic case, defined as

$$
Z_{M B / M J}(\theta)=V \int_{\mathbb{R}^{3}} \exp \left(-\eta_{c / r}(\mathbf{p}) / \theta\right) d^{3} \mathbf{p}
$$

with $V$ the volume and c/r subscripts denoting classical/relativistic expressions for energy in terms of momentum. Note that $\Theta$ has been identified by previous authors [9] [10] [11] as a "relativistic correction factor" and has an asymptotic expansion [14]

$$
\Theta(\theta)^{-1} \sim 1+\frac{15}{8} \theta+\frac{105}{128} \theta^{2}+\mathcal{O}\left(\theta^{3}\right), \quad \theta \rightarrow 0 .
$$

which, as can be seen in figure 2, is a good enough approximation for our uses. This correction factor is of far more general use: it is derived from the normalisation factors of the distributions, so will arise from the ratio of the 
expectation values of any quantity taken over the Maxwell-Jüttner distribution divided by that quantity averaged over the Maxwell-Boltzmann distribution.

Note also, from $\int_{b}^{\infty} z^{n} g(z) d z \geq b \int_{b}^{\infty} z^{n-1} g(z) d z$, where $g(z)$ is any everywhere positive function on $z \geq 0$, we get $P \geq \frac{\eta_{T}}{\theta}$. Therefore we have

$$
R\left(\theta, \eta_{T}\right) \geq \Theta(\theta) \cdot\left[1+\frac{\eta_{T}}{2}\right]
$$

for any cross section and all values of $\theta$ and $\eta_{T}$.

\subsection{Excitation Rate}

Our aim is to derive approximate generic correction factors, dependent on ion species only through the single parameter of threshold energy. We must therefore use a cross section which is a function of only $\eta_{T}$ and $\eta$. For the excitation rate we use the Van Regemorter [12] cross section which for our purposes, if we set the gaunt factor equal in both cases, amounts to

$$
\sigma_{V R} \propto 1 / \eta
$$

Note that we use the classical expression for its energy dependence as the formula was calculated using non-relativistic quantum mechanics. Attempting to adjust the energy dependence of this expression to account for relativity would anyway only give higher-order errors which, given the broad approximations already inherent in using such simple cross sections, can be neglected. Including this in equation 12 gives

$$
P\left[\sigma_{V R}\right]=1+\frac{\eta_{T}}{\theta}
$$

This is plotted in figure 3 .

\subsection{Ionization Rate}

For the ionisation rate we use an empirical formula due to Lotz [13],

$$
\sigma_{L}(x) \propto \frac{\ln (x)}{x}
$$




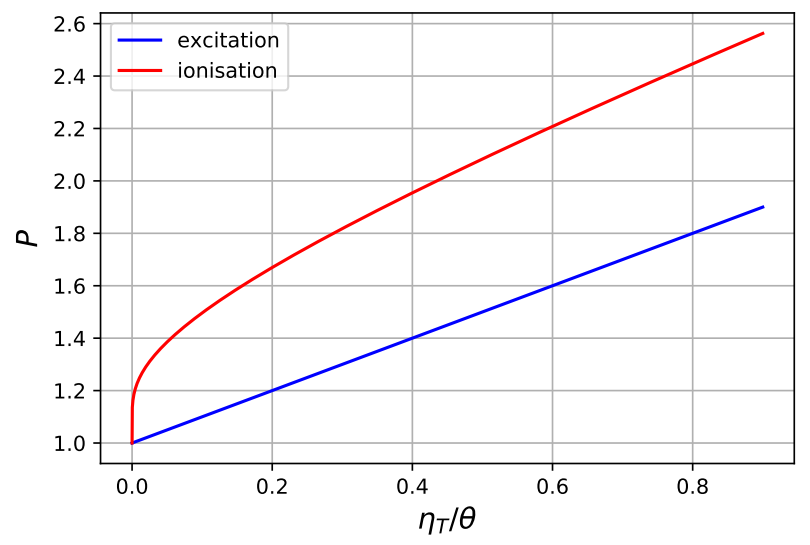

Figure 3: $P[\sigma]$ for excitation and ionisation using, respectively, Van Regemorter's and Lotz' simple expressions for the cross section.

This gives

$$
P\left[\sigma_{L}\right]=\frac{e^{-\frac{\eta_{T}}{\theta}}+E_{1}\left(\frac{\eta_{T}}{\theta}\right)}{E_{1}\left(\frac{\eta_{T}}{\theta}\right)}=1+\frac{e^{-\frac{\eta_{T}}{\theta}}}{E_{1}\left(\frac{\eta_{T}}{\theta}\right)}
$$

where $E_{1}(x)=\int_{x}^{\infty} \frac{e^{-t}}{t} d t$ is the exponential integral. This is plotted in figure 3.

\section{De-excitation and Recombination Rate Corrections}

De-excitation and recombination rates can be calculated from excitation and ionisation using two requirements of equilibrium. The first is the principle of detailed balance, which enforces the requirement that an equilibrium state is constant in time. This gives the reverse rate as proportional to the forwards rate with a ratio that depends on the reactant number densities. The second is that the electrons be distributed between free and bound states in the proportion that maximises total entropy, which is equivalent (under constant temperature and volume) to the minimisation of the Helmholtz free energy, which is in turn equivalent to the maximisation of the total canonical partition function. This affects the reverse rate by determining the reactant number densities. Changing 
the free electron physics by accounting for relativistic effects changes the free electron partition function, and therefore changes the relative entropy of states that have different free electron number, and therefore changes which distribution of the fixed total electron number between the free and bound states maximises the entropy.

This does not apply to excitation processes because the free electron number does not change. This means that relativistic corrections to the de-excitation rate enter only through its proportionality to excitation, and therefore excitation and de-excitation are corrected by the same factor. Ionisation and recombination do change free electron number, so for recombination rates a further correction from adjustments to the free electron entropy is needed. The entropymaximisation requirement for relative occupation of different ionisation states can be expressed by the general Saha equation [15] [16]

$$
\frac{n_{i+1} n_{e}}{n_{i}}=2 Z(\theta) \frac{g_{i+1}}{g_{i}} \exp \left(-\frac{\eta_{i+1}-\eta_{i}}{\theta}\right),
$$

where $n_{i}, g_{i}$ and $\eta_{i}$ are, respectively, the density, degeneracy and reduced energy of ions in the i'th ionisation state and $n_{e}$ is the electron density, and $\mathrm{Z}$ is the appropriate free particle partition function. Using this with detailed

balance $\left(n_{i+1} t=n_{i} r\right.$ with $t$ the recombination rate and $r$ the ionisation), the ratio of recombination rates in the relativistic to classical cases is given

$$
\frac{t_{M J}}{t_{M B}}=\frac{n_{e}^{M J}}{n_{e}^{M B}} \cdot \frac{Z_{M B}(\theta)}{Z_{M J}(\theta)} \cdot \frac{r_{M J}}{r_{M B}}=\Theta(\theta)^{2} \cdot\left[1+\frac{\theta}{2} P\left[\sigma ; \frac{\eta_{T}}{\theta}\right]\right] .
$$

\section{Discussion}

The main results of our calculations can be summarised 


$$
R\left(\theta, \eta_{T}\right)=\left\{\begin{array}{l}
\sqrt{\frac{\pi}{2}}\left[\frac{\sqrt{\theta} e^{-\frac{1}{\theta}}}{K_{2}\left(\frac{1}{\theta}\right)}\right] \cdot\left[1+\frac{\theta}{2}\left(1+\frac{e^{-\frac{\eta_{T}}{\theta}}}{E_{1}\left(\frac{\eta_{T}}{\theta}\right)}\right)\right] \text { ionisation } \\
\frac{\pi}{2}\left[\frac{\sqrt{\theta} e^{-\frac{1}{\theta}}}{K_{2}\left(\frac{1}{\theta}\right)}\right]^{2} \cdot\left[1+\frac{\theta}{2}\left(1+\frac{e^{-\frac{\eta_{T}}{\theta}}}{E_{1}\left(\frac{\eta_{T}}{\theta}\right)}\right)\right] \text { recombination } \\
\sqrt{\frac{\pi}{2}}\left[\frac{\sqrt{\theta} e^{-\frac{1}{\theta}}}{K_{2}\left(\frac{1}{\theta}\right)}\right] \cdot\left[1+\frac{1}{2}\left(\theta+\eta_{T}\right)\right] \quad \text { excitation or de-excitation }
\end{array}\right.
$$

These are plotted in figure 4 for some reasonable values of the threshold energy and temperature. Also plotted is the temperature-dependent prefactor $\Theta(\theta)$ given in equation 13, which has been identified by previous authors [9] [10] [11] as the "relativistic correction factor". It can be seen that our correction factors differ significantly from both this and unity.

The largest possible value of $\eta_{T}$ is for ionisation of hydrogen-like Uranium, with $\eta_{T} \approx 0.23$. In the context of diagnostic dopants in burning ICF plasmas, the reduced ionisation energies of hydrogen-like Krypton and Xenon are around 0.04 and 0.08 respectively. We see in figure 4 that at temperatures around what Tabak predicts could be reached in some realisations of burning ICF [4], we get correction factors of around 0.6-0.8. Relativistic effects appear to be significant in this regime.

\section{Conclusion}

In this paper we have calculated approximate correction factors for ionisation and excitation rates due to collision with free electrons in a plasma to account for special relativity. We have done this by assuming the Van Regemorter expression for the excitation, $\sigma_{V G}(x) \propto 1 / x$, and the Lotz expression for the ionisation cross section, $\sigma_{L}(x) \propto \ln (x) / x$. We then used the Saha equation to extend these results to recombination rates. These correction factors are summarised in equation 23. We also, in section 2.1, found a lower bound for the relativistic correction factor with complete applicability to any cross section. 

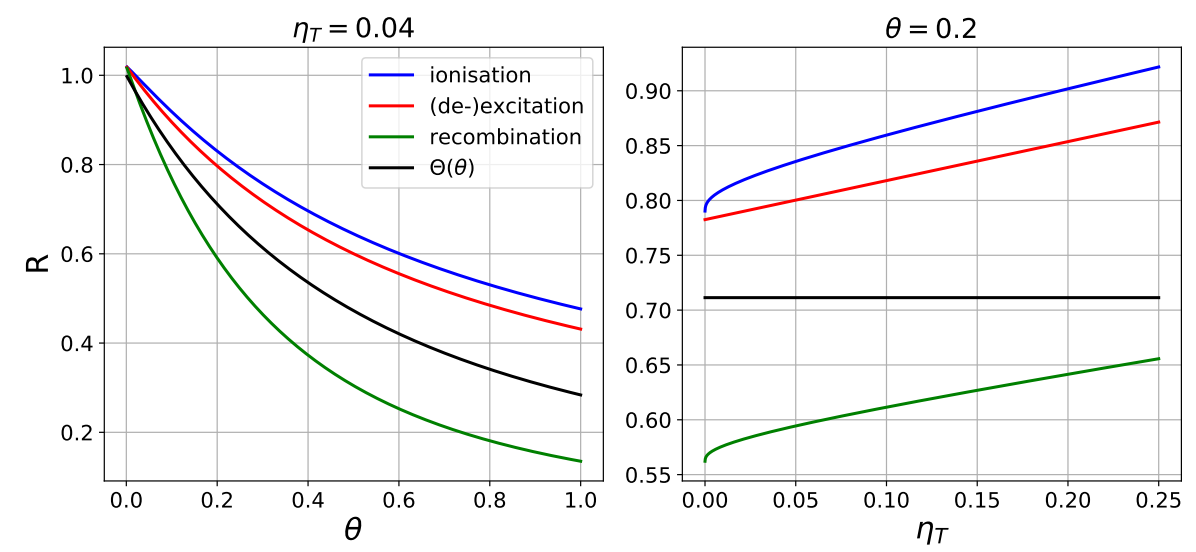

Figure 4: Approximations of the ratio of collisional ionisation, recombination and excitation rates when special relativity is accounted for against when it is not, plotted with sensible values of the reduced threshold energy and reduced temperature. Ionisation and recombination rates are calculated using the Lotz cross section, excitation using Van Regemorter. The temperature-dependent prefactor $\Theta$ is also plotted independently.

Though the precise value of our correction is necessarily limited by the simple forms of the cross sections we have used, there is no reason to expect that the true ratio will be substantially closer to unity. Where we find a ratio far from unity, therefore, it is safe to conclude that accounting for special relativity in the free electron mechanics is important.

\section{Acknowledgements}

This work is supported by the Engineering and Physical Sciences Research Council.

\section{References}

[1] J. Larsen, Foundations of High-Energy-Density Physics, Cambridge University Press, 2017. 
[2] H. A. Scott, Collisional-radiative modeling for radiation hydrodynamics codes, in: Y. Ralchenko (Ed.), Modern Methods in Collisional-Radiative Modeling of Plasmas, Springer International Publishing, Berlin, 2016, Ch. 4, pp. 81-104.

[3] G. J. Tallents, Free electron degeneracy effects on collisional excitation, ionization, de-excitation and three-body recombination, High Energy Density Phys. 20 (2016) 9-16.

[4] M. Tabak, What is the role of tritium-poor fuels in ICF?, Nucl. Fusion 36 (2) (1996) 147-157.

[5] H. Chen, et al., On krypton-doped capsule implosion experiments at the national ignition facility, Phys. of Plasmas 24 (2017) 072715.

[6] W. Biel, et al., Diagnostics for plasma control - from iter to demo, Fusion Eng. Des. (01 2019). doi:https://doi.org/10.1016/j.fusengdes.2018.12.092.

[7] B. W. Smith, et al., Oso-8 x-ray spectra of clusters of galaxies. 1. observations of twenty clusters: Physical correlations - nasa-tm-78097, Tech. rep. (1978).

[8] J. L. Synge, The Relativistic Gas, Series in Physics, North-Holland, 1957.

[9] H. Sampson, D. L. Zhang, C. J. Fontes, A fully relativistic approach for calculating atomic data for highly charged ions, Phys. Rep. 477 (2009) $111-214$.

[10] R. W. B. Best, Clean fusion concepts and efforts - a survey, Nucl. Instrum. Methods Phys. Res. 144 (1977) 1-7.

[11] O. J. Pike, Particle interactions in high temperature plasmas, Ph.D. thesis, Imperial College London (7 2015).

[12] H. Van Regemorter, Rate of collisional excitation in stellar atmospheres, Astrophys. J. 136 (1962) 906-915. 
[13] W. Lotz, Electron-impact ionization cross-sections and ionization rate coefficients for atoms and ions from hydrogen to calcium, Z. Phys. 216 (3) (1968) 241-247.

[14] M. Abramowitz, I. A. Stegun, Handbook of Mathematical Functions: With Formulas, Graphs, and Mathematical Tables, no. 55 in Applied Mathematics Series, Dover Publications, New York, 1965.

[15] M. N. Saha, Liii. ionization in the solar chromosphere, Phil. Mag. 40 (238) (1920) 472-488.

[16] M. N. Saha, On a physical theory of stellar spectra, Proc. R. Soc. Lond. A 99 (697) (1921) 135-153. 